\title{
Size Corrections during Ultrafast Laser Induced Refractive Index Changes in Bulk Transparent Materials
}

\author{
Razvan STOIAN ${ }^{* 1}$, Cyril MAUCLAIR ${ }^{* 1}$, Alexandre MERMILLOD-BLONDIN ${ }^{* 1}$, Nicolas HUOT ${ }^{* 1}$, Eric AUDOUARD ${ }^{* 1}$, \\ Jörn BONSE ${ }^{* 2}$, Arkadi ROSENFELD ${ }^{* 2}$, Ingolf V. HERTEL ${ }^{* 2}$ \\ ${ }^{* 1}$ Laboratoire Hubert Curien, UMR CNRS 5516, Université Jean Monnet 42000 St. Etienne, France \\ E-mail: razvan.stoian@univ-st-etienne.fr \\ ${ }^{* 2}$ Max-Born-Institut für Nichtlineare Optik und Kurzzeitspektroskopie 12489 Berlin, Germany
}

\begin{abstract}
Three-dimensional structuring of bulk dielectric materials usually requires focusing through airdielectric interfaces. Consequently, depth-dependent spherical aberration appears. This determines an elongation of the energy deposition area and restricts the structuring accuracy. We discuss here strategies for counteracting wavefront distortion effects which occur during ultrafast laser induced changes of refractive index. The proposed approaches are based on programmable spatio-temporal pulse shaping and have the objective of concentrating the laser energy on minimal spatial scales. Using adaptive spatial tailoring of ultrashort laser pulses, spherical aberrations can be dynamically corrected, in synchronization with the writing procedure. This facilitates optimal writing of homogeneous longitudinal waveguides over significant lengths. We also show that temporal forming of ultrafast laser pulses restricts the energy spread, leading to a higher confinement. We indicate the role of reduced nonlinearity in plasma formation as a control factor coupling the spatial and temporal response of the material. The size corrections enable higher processing accuracy.

DOI: 10.2961/jlmn.2009.01.0009
\end{abstract}

Keywords: refractive index change, adaptive optics, spatial and temporal pulse shaping

\section{Introduction}

Energy localization via nonlinear absorption led to the successful employment of ultrafast laser radiation for inducing local variations of optical properties in bulk dielectric materials [1]. This has major consequences for generating photonic structures in glasses suitable for optical integration [2]. Specifically, ultrashort pulse lasers were employed in embedded waveguide writing techniques which rely on positive refractive index variations in the laseraffected region [3-5]. Waveguiding structures were obtained in different optical materials by translating a focused laser beam longitudinally or transversally with respect to the propagation axis. Different beam shaping schemes were used to preserve a certain symmetry of writing [6-11]. Nevertheless, focusing through an air-dielectric interface causes detrimental wavefront distortions. Refraction of optical rays at the interface induces an aperture and depthdependent elongation of focal spots. A schematic representation of the focus distortion due to spherical aberration is depicted in Fig. 1. This is responsible for a lower energy density value [10] and a modulation in the axial intensity profile $[11,12]$. This fact has dramatic effects for energy propagation in the processed zones and for the morphology and homogeneity of the laser-induced structures [13-15].

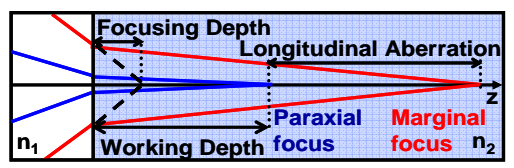

Fig. 1 Description of spherical aberration generated by focusing through air-dielectric interfaces.

The consequences of aberrated beams for photo-written waveguides are manifold: low processing accuracy, in- creased losses, inhomogeneous refractive index modulation, or the apparition of non-guiding domains. The employment of dynamic procedures for aberration corrections enables transporting the laser energy to the impact point without significant spatial dispersion. We describe here a procedure using adaptive spatial and temporal pulse shaping to achieve a spatial compensation function and to restrict the structure elongation, determining higher process precision.

The effect of spherical aberration in ultrafast laser material processing was previously analyzed via depthdependent material modification thresholds and aspectratio measurements, underlining the importance of preserving excitation conditions [13-17]. Microscope objective collars delivering adjustable compensation of spherical aberration were employed for controlling the modification size in bulk optical materials [12]. Corrective functions using adaptive optics were applied to minimize depthdependent aberrations [17] for data storage and microscopy applications. Consequences for quality waveguide writing were already indicated [12-17]. With emphasis on applications in waveguiding technologies, we first review here a technique able to dynamically correct spherical aberration and to optimize the process of photowriting longitudinal waveguides in a dielectric environment using automated spatial phase filtering integrated in adaptive loops. This is based on spatial phase retardation introduced by programmable optical modulators responding to a feedback derived from the laser action, being driven by a global optimization strategy. Even though the problem of spherical aberration can be analytically addressed using Zernike-polynomial decomposition, this requires calibration of the phase retardation induced by the shaping system and an accurate correspondence between the phase-manipulation plane and the 
pupils of the optical focusing system. We intend to show that global search algorithms [18] represent an effective calibration-free technique, able to significantly improve the structuring process even when the nature of wavefront deformation is not accurately known. Advantages for material structuring applications may be derived when the feedback response is obtained directly from a laser processing result.

However, if the structure size is primarily determined by spherical aberration, the modulation of the material response is regulated by nonlinear propagation effects. From an alternative perspective the nonlinear aspect of interaction may also suggest the temporal form as a control knob to achieve energy confinement. Adaptive control of pulse temporal forms was recently used to regulate filamentary propagation in nonlinear environments $[19,20]$. The location and the spectral properties of the ionization region were shown to be modulative. The key factor is the intensity feedthrough which determines the competition between self-focusing and ionization. Breakdown probability was also observed to be controllable via temporal envelopes [21]. At surfaces, manipulation of pulse frequencies indicated sensible variations in the damage threshold [22]. Asymmetric intensity envelopes have also shown surprising reduction in the damaged area, below the diffraction limit [23]. The balance between photo and collisional ionization mediates the localized formation of a hot electron population, taking into account the different process dependencies on intensity and wavelength. All these observations indicate flexibility in manipulating propagation, ionization and energy gain events generated by ultrashort laser pulses in nonlinear environments using judicious temporal intensity adjustments. With a focus on refractive index modification processed in optical materials we address below some aspects generating spatial energy confinement and structural modification in dielectric media by suitable temporal pulse forming when the illuminated zone is elongated due to spatial wavefront distortions. A programmable temporal shaping is used.

This work reviews some recent results concerning the possibility of structure size confinement using spatial and temporal pulse manipulation. The paper is organized as follows. The experimental section provides an overview of the achieved spatio-temporal flexibility in irradiation, the detection method, and the subsequent adaptive loops. The discussion section describes the impediments posed by spherical aberration in reaching regimes of refractive index increase. The results of adaptive spatial correction are evaluated with respect to the possibility to induce symmetric channels of high-contrast positive index changes over long distances. Finally, the use of temporal pulse forming and nonlinearity control for attaining superior energy confinement will be discussed as well.

\section{Experimental setup and methods}

\subsection{Irradiation and refractive index detection setup}

Parallelepipedic glass samples (fused silica $a-\mathrm{SiO}_{2}$ and borosilicate crown BK7) are irradiated with $150 \mathrm{fs}$ and 160 fs pulses from $800 \mathrm{~nm}$ Ti:sapphire ultrafast lasers systems at various repetition rate between single isolated pulses and $100 \mathrm{kHz}$. The laser beam was focused inside the target by a microscope objective (numerical aperture 0.45 , working distance $17 \mathrm{~mm}$ ). To estimate the results of irradiation, a fast microscopy method is employed. Detection of the structures is realized online by optical phase-contrast microscopy (PCM). The method allows monitoring relative changes in the refractive index, realizing a side view twodimensional map of the phase object. Positive or negative optical phase changes relative to the background can be evaluated based on the image gray-value shift. Correspondingly, dark regions denote positive index changes, while the light zones indicate a negative refractive index variation or the presence of light scattering centers. The material modification profiles can thus be precisely evaluated.

\subsection{Adaptive spatio-temporal design for laser pulses}

The laser system incorporates a programmable liquidcrystal pulse-shaping apparatus, which realizes temporal pulse tailoring using spectral phase filtering [24]. This device has the role to manipulate the spectral frequency components of the pulse, allowing for spectral phase modulation and subsequent pulse temporal design. Additionally, the laser system incorporates at the output, within the pulse control unit, a programmable, optically-addressed twodimensional beam-forming system which performs spatial pulse tailoring using spatial phase control of the incident laser pulse [25]. The external spatial light modulator comprises an optically-addressed liquid-crystal light valve, which is imaged onto the focusing system used for material processing. As a result, the output laser beam can be tailored spatially and temporally in a flexible way to quasiarbitrary intensity profiles.

A feedback loop connects the microscopy detection and the pulse control unit, being guided by adaptive optimization algorithms of genetic character. The optimization mechanism was described in previous works (see e.g. [26] and references therein). The pulse tailoring unit performs the variation of the incoming intensity either in the temporal or in the spatial domains and the detection of the refractive index delivers the quantitative evaluation of the laser action. Retrieving the 2D map of the photo-inscribed object, an objective functional is defined by analyzing the axial morphology of the photo-inscribed phase object and comparing it with a user-designed profile. Based on the resemblance a note (fitness) is assigned. The laser pulse envelope is iteratively changed to increase the success of particular laser pulses in maximizing of the functional fitness. For each optimization attempt, particular functional objectives will be discussed. The optimal outcome is an intensity shape that produces index patterns close to desired profiles.

\section{Results and discussion}

\subsection{Static and dynamic regimes of laser processing}

For demonstration purposes we have chosen Schott BK7 borosilicate crown glass as a model material due to potential applications in optics and microfluidics. A second reason is related to the fact that this material shows a narrow laser processing window for positive refractive index changes $[3,27]$. This requires fine tuning of the energy density which enhances the demands for precise processing and, therefore, for preserving excitation conditions at arbitrary depths. High energy concentrations can generate positive refractive index changes based on material compaction, 
thus enabling guiding using this regime of photoinscription. Wavefront distortions have then consequences for writing performant waveguides of increased dimensions, leading to the challenge of preserving energy densities and positive index changes at arbitrary distances.

To quantify the effect of laser irradiation under optimal focusing conditions we evaluate the results of structuring in the vicinity of the surface, where the influence of spherical aberrations is minimal. Fig. 2 (a-d) shows the result of irradiation of $150 \mathrm{fs}$ pulses at a working depth of $200 \mu \mathrm{m}$ for two different input average powers, $125 \mathrm{~mW}$ and $80 \mathrm{~mW}$ at $100 \mathrm{kHz}$. Parts (a) and (b) show the static refractive index modifications produced by $10^{5}$ pulses. The fs irradiation induces a dominant refractive index decrease denoted by the white color. In case of the high energy static structures, a shallow region of positive index change (black color) surrounds the low index core, terminating with an elongated trace of high index material at the structure tip. The low energy preserves somehow the topology with the region of surrounding compression being drastically reduced.

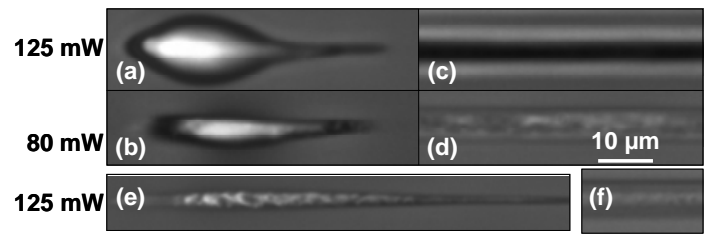

Fig. 2 Static [left, $(a, b)]$ and dynamic, longitudinally written [right, $(c, d)$ ] PCM modifications induced in BK7 by ultrafast laser radiation at different input powers and at a depth of $200 \mu \mathrm{m}$ behind the airdielectric interface. The static irradiation corresponds to $10^{5} \mathrm{pulses} / \mathrm{site}$ while the dynamic structures are made at a scanning speed of $1 \mu \mathrm{m} / \mathrm{s}$ at $100 \mathrm{kHz}$. Laser pulses are incident from the left. Waveguide writing conditions are achieved at high incident powers. (e,f) static and dynamic traces in aberrated conditions (depth $3 \mathrm{~mm}$ ).

This specific topology can presumably be connected to a strong expansion of the irradiated volume in glasses such as BK7, characterized by high thermal expansion. After the initial laser heating, the material expands while cooling, which, in turn, inhibits the backward relaxation and quenches the material in a low density phase. However, high energy densities generate compressive shock waves [28] and determine the formation of a strongly compacted region around the low density core. These provide the prerequisites for a significant axial positive change in the refractive index at the structure tip. Upon photoinscription, if the energy concentration stays sufficient, the high density positive index phase can be replicated during the scan, leading to the formation of a waveguide. In order to observe the consequence for a dynamic regime of photoinscription and to create guiding elements, the focal point was rastered along the irradiation axis. Same irradiation conditions were used to scan the beam longitudinally in a spatial region located around the working depth of $200 \mu \mathrm{m}$. The scan direction was towards the laser beam, with the starting point into the bulk. Fig. 2 (c,d) indicates structures written longitudinally at a scanning speed of $1 \mu \mathrm{m} / \mathrm{s}$, corresponding to the situations (a) and (b). For the high power longitudinal line, a contrasted region of positive index change is visible at high energies, denoted by the intense black color, bordered by narrow lines of decreased index. This structure shows a high and homogeneous index contrast. The low energies correspond to a negative refractive index change (represented by the dominant white color), which, in normal conditions, inhibits guiding. The index variation is not uniform, with an alternation of positive and negative changes. It appears from the figure that a uniform region of positive refractive index change occurs during translation only when a critical density of energy was transported at the interaction place. This indicates the achievement of a high temperature in the interaction region, followed by the onset of the surrounding compressed region. The longitudinal translation at high repetition rates leads to a high density trace upon scanning in the direction of the laser pulse. To reach dynamic positive index change regime, a power threshold of approximately $90 \mathrm{~mW}$ was found necessary in our experimental conditions. The transition power depends as well on the scan velocity. Below this value, moderate thermal expansion and rarefaction upon cooling determine to a large extent the material response, inducing a dominant low density low index phase. Inevitably upon irradiation at different depths, the energy density decreases due to spherical aberration and limits the possibility to trigger the positive index regime far from the airglass interface. This is shown in Fig. $2(e, f)$ for structures induced by $125 \mathrm{~mW}$ at $100 \mathrm{kHz}$ at a depth of $3 \mathrm{~mm}$. Consequently, we have corrected the spatial phase distortion using the above-mentioned spatial-domain optimization loop in order to preserve a high energy density.

\subsection{Adaptive spatial correction of wavefront distor- tions and processing solutions}

We noted above that high energy, low density structure replication via scanning delivers a positive refractive index change. This may be connected to the presence of the surrounding high index, compressed region. Since this property degrades with depth, we will focus below on the possibility to annihilate wavefront distortions effects and to restrict the energy spread by spatial phase adjustments. In order to reach the compressive regime at arbitrary depths, it is imperative that the energy delivery remains concentrated to the narrowest region. This was achieved by adaptively determining the corrective phase masks in the microscopybased feedback loop presented before [26].

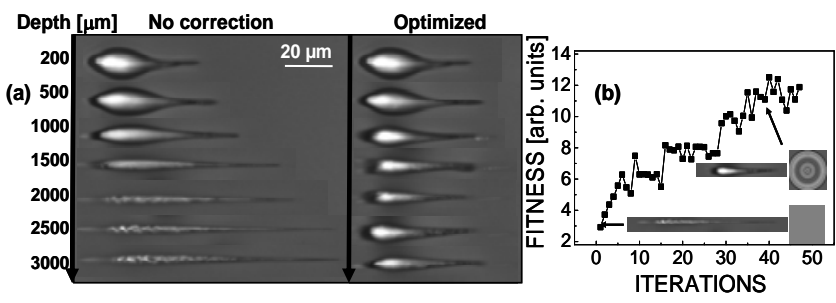

Fig. 3 (a) Non-corrected (left) and spatially-corrected (right) static PCM structures induced at different depths. The structures are generated by $10^{5}$ pulses of $150 \mathrm{fs}$ duration at $100 \mathrm{kHz}$ and $125 \mathrm{~mW}$ average power. (b) Evolution of the trace fitness during the optimization run at the depth of $2500 \mu \mathrm{m}$. Example of traces and corresponding gray-level phase masks at different moments are given as well.

In the case of spatial correction of aberration, the feedback fitness is determined by comparison to the unaber- 
rated structure and is related to the inverse of the trace length. In order to avoid trivial solutions which are highly energy dispersive and lead to damage on limited regions (where the threshold was surpassed), an additional condition regarding the contrast of the phase shift in the structure was added. Structure length is determined by the sum of pixels that have a gray-value different from the average background. The minimization of the trace longitudinal size defines the success of the irradiation sequence. Optimization runs were effectuated at different depths from 500 $\mu \mathrm{m}$ to $3000 \mu \mathrm{m}$ in steps of $500 \mu \mathrm{m}$. The limiting factor is imposed by the objective working distance. Fig. 3 (a) shows the results of the optimization procedure for photoinscription as compared to the effect of the uncorrected pulse for different depths into the glass material. An example of the iterative improvement during the optimization run is given in Fig. 3 (b). If the structures induced by the uncorrected pulse show a threefold lengthening down to a depth of $3000 \mu \mathrm{m}$ [Fig. 3 (a) left] the correction procedure has stabilized the structure length at almost the initial size [Fig. 3 (a) right]. Nevertheless, if the structure size is kept at a constant level, the energy density is slightly decreased as compared to the unaberrated structures, as indicated by the level of the white contrast [Fig. 3 (a) right]. Consequently, the bulk excitation density will still be below the threshold for inducing a positive refractive index change during the longitudinal writing in the profound regions. Since the laser source has limited energy output which is just above the transition threshold, only $10 \%$ size variation with respect to the unaberrated trace will decrease the energy density below the critical value. To compensate for this slight deviation with respect to the unaberrated structure and due to the limitations in the input laser power in our case, additional corrective solutions are required. A complementary technique for increasing the energy density deposited within the material is represented by pulse linear temporal chirping for the following reason. Ultrafast irradiation focused with moderate to high NAs results in structures where self-focusing is dominated by light defocusing on laser-induced electron-hole plasmas. To minimize the effect of light spreading due to defocusing, the moment of reaching the maximum plasma density has to be delayed with respect to the beginning of the laser pulse. A longer temporal intensity envelope and a retarded plasma formation produce a less effective defocusing, allowing the energy to be efficiently concentrated in the irradiated region.

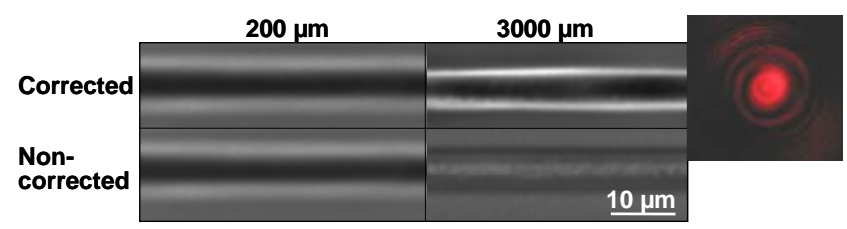

Fig. 4 Longitudinal structures (PCM) at different working depths in corrected (top) and noncorrected (bottom) cases. The corrections enable a positive refractive index change over a distance of $3 \mathrm{~mm}$. Scanning speed is $1 \mu \mathrm{m} / \mathrm{s}$ at $125 \mathrm{~mW}$ average power. Right, far-field pattern of the guided mode at $633 \mathrm{~nm}$ for the corrected guide.

The success of the operation is verified by effectively writing longitudinal guiding structures. Combining the spatial correction with a gradual increase in pulse duration with depth (up to $2.7 \mathrm{ps}$ at $3 \mathrm{~mm}$ by programmable second order dispersion) allows writing waveguiding structures as long as $3 \mathrm{~mm}$ for relatively tight focusing. To avoid catastrophic damage during the longitudinal scan towards the front surface, the pulse was continuously compressed, reaching the shortest value at a depth of just below $1 \mathrm{~mm}$. A positive quadratic dispersion coefficient was found to be slightly more effective in obtaining the positive index change regime. The spatial phase correction masks were as well applied in synchronization with the advance of the structure inside the glass material. A linear interpolation between successive corrective solutions at various depths was used to generate new patterns to be gradually applied at the scan speed of $1 \mu \mathrm{m} / \mathrm{s}$ [26]. The photoinscription outcome is depicted in the top part of Fig. 4 which synthesizes the result of the dual corrective procedure. Both ends of the longitudinal structure are shown. A uniform dark structure is becoming visible, indicating a positive index change all along the trace length. This shows that the spatio-temporal correction enables the generation of a uniform cylindrical waveguide with a high positive index contrast for a length superior to standard irradiation and which allows symmetric guiding. By evaluating the numerical aperture of the generated guide at $633 \mathrm{~nm}$, a relative index increase of approximately $5 \times 10^{-3}$ was estimated. For comparison, the uncorrected trace is shown in the bottom part of Fig. 4. In this case, the guiding region is restricted to less than $1 \mathrm{~mm}$, the rest of the trace showing a negative index change.

\subsection{Temporal control of structure sizes}

We discuss below the effect of wavefront distortions in fused silica [29]. We have mentioned before, that, within the longitudinal aberration, modulation of the refractive index may appear due to nonlinear propagation effects. Phase-contrast observations of permanent structures induced by a single short laser pulse $(160 \mathrm{fs}, 1 \mu \mathrm{J})$ in fused silica are presented in Fig. 5 for various working depths.

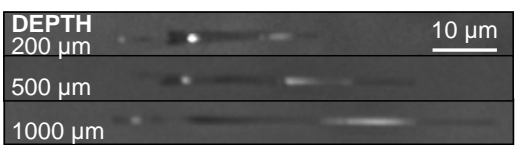

Fig. 5 PCM images of permanent structures induced by single short pulses in bulk irradiated $a-\mathrm{SiO}_{2}$ for various depths. The laser pulse is coming from left. Irradiation parameters: $160 \mathrm{fs}$ and $1 \mu \mathrm{J}$.

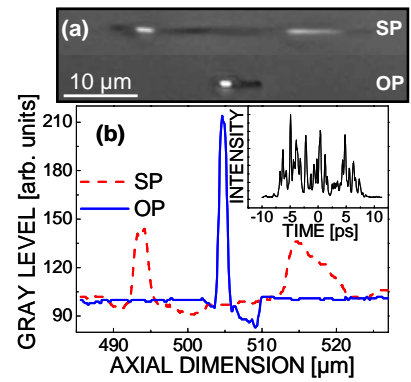

Fig. 6 (a) Short 160 fs (SP) and optimal pulse (OP) structures in $a-\mathrm{SiO}_{2}$ at a depth of $500 \mu \mathrm{m}$ and 1.3 $\mu \mathrm{J}$ energy. (b) Corresponding refractive index axial cross-sections. The inset shows the optimal pulse.

The permanent traces indicate a refractive index modulation which is generated by an axial variation of excitation. 
This modulability derives from a dynamic filamentation balance between dispersion, nonlinear focusing, and ionization, resulting in a varying refractive index structure along the propagation axis. This shows a central positive (dark) index change bordered by white regions of decreased density and increased inhomogeneity. The white domains were previously identified with regions of maximum energy deposition [30], where material expands thermally. The left-side white dot is presumably a self-focusing effect [3032]. The black, positive refractive index region involves a complex mixture of thermomechanical phenomena and defect generation. If the spatial index modulation accentuates with the depth, the modulation domain appears to be defined by the longitudinal aberration [Fig. 1] [10].

Preserving positive index changes, we have attempted to reduce the spatial extent of the white regions using the temporal shaping strategy. The objective functional was defined accordingly. The microscopy image analysis determines the area of positive (negative) index change by summing the number of black (white) pixels detectable above the noise threshold on the PCM image within a narrow axial region. The optimization functional is defined by the ratio between the number of black and white pixels, respectively. We have applied the automated temporal feedback loop with the purpose of maximizing the index ratio. The optimization result is an intensity shape that has produced refractive index patterns with maximum contrast. The result of the optimal pulse (OP) compared to the $160 \mathrm{fs}$ short pulse (SP) irradiation is shown in Fig. 6 for $1.3 \mu \mathrm{J}$ input energy and $500 \mu \mathrm{m}$ depth. The optimal sequence takes the form of a structured intensity envelope extending on a picosecond scale. This particular sequence provides a more than two times increase for the objective functional. The control parameter appears to lie in the extended envelope. At the same time, a drastic decrease in the size of the structure is noticeable, while keeping the negative index region to a minimum. The consequences of a ps envelope are twofold [30]. Firstly, the ps envelope induces a retarded, low density, spatially-modulated plasma. This creates a smaller negative shift for the incoming energy and less defocusing, helping thus to concentrate the energy in the region of best focus. Secondly, the nonlinearity of excitation diminishes, allowing efficient absorption only in a restricted region around the geometric focal point. It should be mentioned that, with suitable pulse temporal shapes, size reduction can be observed even for traces at $200 \mu \mathrm{m}$ depth (Fig. 7), where the aberrations effects are minimal.

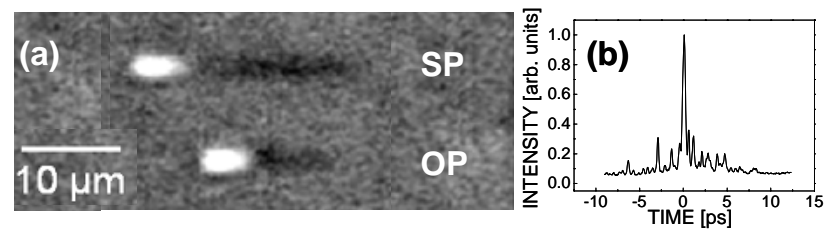

Fig. 7 (a) SP and OP induced structures at a depth of $200 \mu \mathrm{m}$ and $0.4 \mu \mathrm{J}$ input energy in $a-\mathrm{SiO}_{2}$. (b) The corresponding OP intensity shape.

The nonlinear propagation coupled with the material response defines the topology of the solution space. In order to gain insights into the propagation and modification factors we have attempted to simplify the control landscape by using less complex pulse shapes, namely temporally broadened pulses and double pulse sequences with variable separation times. The long envelopes and peaked structures were features observed in the optimal solution. The irradiation energy was varied as well. The results are given in Fig. 8. The variation of the pulse duration at $1 \mu \mathrm{J}$ [Fig. 8 (a)] indicates the following behavior. For moderate stretching, below $6 \mathrm{ps}$, the energy concentrates efficiently, leading to the onset of a hot spot in the central area. The main modification is a negative index change due to efficient heating and thermal expansion. However, beyond a value of $6 \mathrm{ps}$, the behavior changes. The positive index change takes over, with a gradual reduction of the low index region. The double pulse result [Fig. 8 (b)] shows mainly the rarefaction effect at high energy concentration and indicate that absorptive phases are present in between the pulses.

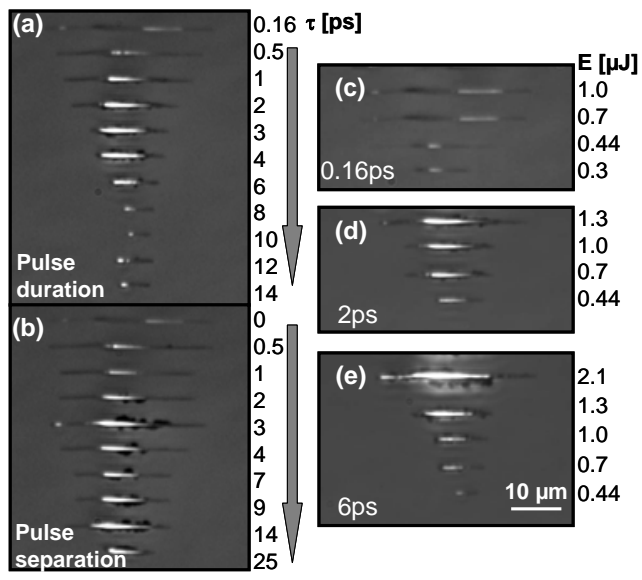

Fig. 8 Structures induced by pulses with (a) different durations or (b) double pulse sequences with variable separations at $1 \mu \mathrm{J}$. Energy effects for pulses with selected durations; (c) short 0.16 ps pulse, (d) 2 ps pulse, and (e) 6 ps pulse. All structures are made in $a-\mathrm{SiO}_{2}$ at a depth of $500 \mu \mathrm{m}$.

The subsequent question relates to the physical factor for reducing the modification size, despite the geometrical spread of the focal area. The imposed dispersion generates a complex temporal pulse shape at the focus which cannot be determined accurately. We therefore concentrate on qualitatively observing the experimental results. This brings interesting clues for explaining the partial filamentation control and proposing probable bulk excitation paths. Following the wavefront distortion, the laser beam is first focused at the paraxial location (closer to the surface). If a low density plasma is formed, a small defocusing effect is induced, shifting the energy deposition further away from the interface. Additionally, the lower intensity ensures that ionization takes place on a reduced scale, with a change in morphology at low excitation densities. As an alternative way to vary the interaction nonlinear character we have subsequently reduced the energy [Fig. 8 (c-e)] for pulses with different durations to observe the alterations in the index modulation length and the effect on the propagation nonlinearity at the respective pulse durations. An increase of the modification threshold was observed, ranging from approximately $0.09 \mu \mathrm{J}$ for the short pulse to $0.6 \mu \mathrm{J}$ for 14 ps pulse (not shown). This indicates the increasing energy costs for longer pulses as a consequence of reduced absorption nonlinearity. Structures with dominant highlycontrasted positive index changes at low energies can be 
obtained for the short pulses or for pulses exceeding a duration of 6 ps in a large energy domain around the modification threshold. This particular aspect shows that the threshold criterion by itself is not sufficient to explain the optimization results and that the plasma mediates spatially the energy deposition process. This allows distributing the input energy in precise doses in the spatial and temporal domains. Thus, the nonlinearity control regulates selffocusing and, as well, the energy requirements together with a plasma delayed energy loss.

\section{Conclusions}

We have firstly shown that adaptive optics in the spatial domain in connection to feedback loops are effective means to fulfill corrective functions during ultrafast laser photoinscription of waveguiding structures in optical glasses. We concentrated here on a procedure of wavefront correction, namely spherical aberration, which can be correlated with the photoinscription process, requires no calibration, and allows extended versatility. The spatial correction method was accompanied by pulse temporal stretching to allow efficient energy confinement. Increased precision for $3 \mathrm{D}$ processing and long waveguide writing lengths were obtained, leading to a visible improvement of the structuring process. This enabled the generation of homogeneous longitudinal waveguides over long distances even in tighter focusing conditions. Preserving the energy density irrespective of the processing depth allows triggering mechanisms necessary to generate highly-contrasted positive refractive index change, mandatory for low-loss guiding objects. Alternatively, adaptive temporal shaping induces spatio-temporal pulse behaviors capable of localizing energy on limited spatial scales, determining as well a dominant region of refractive index increase. The decreased nonlinearity and the lower ionization efficiency assist the energy confinement while reducing the structure ellipticity.

\section{Acknowledgments}

ANR and PICS programs are gratefully acknowledged.

\section{References}

[1] E. N. Glezer and E. Mazur, Appl. Phys. Lett. 71, (1997) 882.

[2] K. M. Davis, K. Miura, N. Sugimoto, and K. Hirao, Opt. Lett. 21, (1996) 1729.

[3] V. R. Bhardwaj, E. Simova, P. B. Corkum, D. M. Rayner, C. Hnatovsky, R. S. Taylor, B. Schreder, M. Kluge, and J. Zimmer, J. Appl. Phys. 97, (2005) 083102

[4] D. Ehrt, T. Kittel, M. Will, S. Nolte, and A. Tünnermann, J. Non-Cryst. Solids 345-346, (2004) 332.

[5] Y. Liu, H. Jiang, Q. Sun, Z. Wu, H; Yang, and Q. Gong, J. Opt. A: Pure and Appl. Opt. 7, (2005) 198.

[6] G. Cerullo, R. Osellame, S. Taccheo, M. Marangoni, D. Polli, R. Ramponi, P. Laporta, and S. De Silvestri, Opt. Lett. 27, (2002) 1938

[7] V. Diez-Blanco, J. Siegel, A. Ferrer, A. Ruiz de la Cruz, and J. Solis, Appl. Phys. Lett. 91, (2007) 051104

[8] R. R. Thomson, A. S. Bockelt, E. Ramsay, S. Beecher, A. H. Greenaway, A. K. Kar, and D. T. Reid, Opt. Express $16,(2008) 12786$.
[9] Y. Cheng, K. Sugioka, K. Midorikawa, M. Masuda, K. Toyoda, M. Kawachi, and K. Shihoyama, Opt. Lett. 28, (2003) 55.

[10] N. Huot, R. Stoian, A. Mermillod-Blondin, C. Mauclair, and E. Audouard, Opt. Express 15, (2007) 12395.

[11] M. J. Booth, M. A. A., Neil, and T. Wilson, J. Micros. 192, (1998) 90

[12] C. Hnatovsky, R. S. Taylor, E. Simova, V. R. Bhardwaj, D. M. Rayner, and P. B. Corkum, J. Appl. Phys. 98, (2005) 013517.

[13]A. Marcinkevicius, V. Mizeikis, S. Juodkasis, S. Matsuo, and H. Misawa, Appl. Phys. A 76, (2003) 257.

[14] Q. Sun, H. Y. Jiang, Y. Liu, Y. Zhou, H. Yang, and Q. Gong, J. Opt. A: Pure and Appl. Opt. 7, (2005) 655.

[15]D. Liu, Y. Li, R. An, Y. Dou, H. Yang, and Q. Gong, Appl. Phys. A: Mater Sci. Process. 84, (2006) 257.

[16] M. Ams, G. D. Marshall, P. Dekker, M. Dubov, V. K. Mezentsev, I. Bennion and M. J. Withford, IEEE J. Sel. Top. Quant. Elec. 14, (2008) 1370.

[17] M. J. Booth, M. Schwertner, T. Wilson, M. Nakano, Y. Kawata, M. Nakabayashi, and S. Miyata, Appl. Phys. Lett. 88, (2006) 031109.

[18] J. Hahn, H. Kim, K. Choi, and B. Lee, Appl. Opt. 45, (2006) 915.

[19] G. Heck, J. Sloss, and R. J. Levis, Opt. Commun. 259, (2006) 216.

[20] R. Ackermann, E. Salmon, N. Lascoux, J. Kasparian, P. Rohwetter, K. Stelmaszczyk, S. Li, A. Lindinger, L. Wöste, P. Béjot, L. Bonacina, and J. -P. Wolf, Appl. Phys. Lett. 89, (2006) 171117.

[21] M. Y. Shverdin, S. N. Goda, G. Y. Yin, and S. E. Harris, Opt. Lett. 31, (2006) 1331.

[22] E. Louzon, Z. Henis, S. Pecker, Y. Ehrlich, D. Fisher, M. Fraenkel, and A. Zigler, Appl. Phys. Lett. 87, (2005) 241903.

[23] L. Englert, B. Rethfeld, L. Haag, M. Wollenhaupt, C. Sarpe-Tudoran, and T. Baumert, Opt. Express 15, (2007) 17855.

[24] A. M. Weiner, Rev. Sci. Instrum. 71, (2000) 1929.

[25] N. Sanner, N. Huot, E. Audouard, C. Larat, C., J. -P. Huignard, and B. Loiseaux, Opt. Lett. 30, (2005) 1479.

[26] C. Mauclair, A. Mermillod-Blondin, N. Huot, A. Audouard, and R. Stoian, Opt. Express, 16 (2008) 541.

[27] A. Mermillod-Blondin, I. M. Burakov, Yu. P. Meshcheryakov, N. M. Bulgakova, E. Audouard, A. Rosenfeld, A. Husakou, I. V. Hertel, and R. Stoian, Phys. Rev B 77, (2008) 104205.

[28] M. Sakakura, M. Terazima, Y. Shimotsuma, K. Miura, and K. Hirao, Opt. Express 15, (2007) 5674.

[29] A. Mermillod-Blondin, A. Rosenfeld, J. Bonse, I. V. Hertel, C. Mauclair, E. Audouard, and R. Stoian, Appl. Phys. Lett. 93, (2008) 021921.

[30] I. M. Burakov, N. M. Bulgakova, R. Stoian, A. Mermillod-Blondin, E. Audouard, A. Rosenfeld, A. Husakou, and I. V. Hertel, J. Appl. Phys. 101, (2007) 043506.

[31] C. B. Schaffer, A. O. Jamison, and Eric Mazur, Appl. Phys. Lett. 84, (2004) 1441.

[32] L. Sudrie, M. Franco, B. Prade, A. Mysyrowicz, Opt. Commun. 191, (2001) 333.

(Received: July 8, 2008, Accepted: March 4, 2009) 